\title{
Highly selective transmission and absorption from metasurfaces of periodically corrugated cylindrical particles
}

\author{
Zhenisbek Tagay and Constantinos Valagiannopoulos \\ Department of Physics, School of Science and Technology, Nazarbayev University, 53 Qabanbay Batyr Avenue, \\ Astana, KZ-010000, Kazakhstan
}

(Received 7 May 2018; published 25 September 2018)

\begin{abstract}
Gratings of infinite wires make, perhaps, the simplest class of metasurfaces, which, however, are utilized for a variety of different objectives in wave manipulation. Importantly, due to their analytical solvability, they can be fully optimized in a fast and direct way. In this study, a lattice of periodically corrugated cylindrical particles under oblique plane-wave excitation is considered and treated rigorously. Several cases of particle radii, distances between two consecutive cylinders, and angles of illumination are examined; as a result, very high selectivity of the metasurface response in terms of line-of-sight transmission and absorption is reported. That abrupt change in the device output becomes even more dramatic in the parametric vicinity of the emergence of new refractive orders, which makes the proposed metasurface exceptionally fitting for switching, filtering, and sensing applications.
\end{abstract}

DOI: 10.1103/PhysRevB.98.115306

\section{INTRODUCTION}

If metamaterials mimic exotic electromagnetic properties not findable in nature, metasurfaces [1] emulate exotic boundary conditions admitting general beam steering and field transformation. Indeed, placing multiple similar objects across a surface can change the characteristics (phase, amplitude, and polarization) of the waves passing through it in an arbitrary way [2]. These applications and functionalities cover a broad range of operational wavelengths from microwaves to visible [3] and may also concern nonelectromagnetic interactions like acoustic [4] ones. Introducing phase discontinuities within the thin parallelepiped volume of a metasurface separating two media allowed the reformulation of the laws of reflection and refraction by applying Fermat's principle [5] and revealed unprecedented opportunities for light manipulation with planar photonics [6]. Highly efficient designs have been proposed, including polarization converters with help from plasmonic nanoslits [7] and lattices of dielectric elliptical nanoposts that can control the wave phase with subwavelength spatial resolution [8]. Furthermore, successful beam focusing has been experimentally demonstrated by employing patterned layers of silicon on quartz substrates [9], while nonplanar gratings of nanoantennas can conceal three-dimensional arbitrarily shaped objects by wrapping around them [10]. Reflections along multiple directions from metasurfaces can also formulate multiport network analogs [11] with scattering matrices corresponding to power splitters, isolating mirrors, or other nonreciprocal devices.

Periodic gratings of cylinders make, perhaps, a less complex class of electromagnetic metasurfaces since their constituent elements are parallel rods of circular (or other) cross section, which render the structure two-dimensional. However, despite their simplistic nature, metasurfaces of cylindrical particles have been proven to be very effective for various operations since they are the two-dimensional equivalent of photonic crystals with their countless applications [12].
Highly directional emission from an aperture with periodic metallic grooves has been observed [13], while gradient metasurfaces of alternating rectangular dielectric rods can support perfect anomalous reflection of visible light [14]. Gratings of parallel wires can also serve as ultraefficient absorbers by engineering their particles to work like passive Huygens sources [15] or leaky-mode waveguides [16]. Finally, lattices of circular rods can give a greatly enhanced magnetic field for magnetic resonance imaging applications [17], while nonscattering arrays of corrugated cylinders can be constructed to make perfect antenna radomes [18].

Apart from their multifunctionality, metasurfaces of parallel cylinders are, in most cases, analytically solvable using either canonical expansions of wave equation solutions or integral equation formulations [19]. In the case of particles with a circular cross section, the problem has been extensively studied for many decades in several ways. The followed approaches include connecting the multiple-scattering amplitude with that of an isolated element [20], implementing a lattice sum technique [21], performing multipole/plane-wave expansions [15,22], and applying a direct Neumann iteration procedure [23]. Even when the cylinders have a noncircular shape, rigorous treatment is still feasible with the use of integral equation methodologies employing grids of triangular elements [24] or entire-domain basis functions [14]. Having a closed-form analytical solution utilizing the aforementioned techniques is not only sophisticated but also very useful when testing many different grating designs. Indeed, analytical formulas can substantially lighten the computational burden accompanying structural or textural optimization, compared to running multiple full-wave simulations via commercial packages.

In this work, we consider cylindrical particles periodically corrugated along their axis, made of only two materials whose proportion changes linearly with respect to radial dimension. In fact, we use the concept of digital metamaterials [25] but in 
a configuration which avoids abrupt texture changes. In order to render our problem analytically solvable and acquire all the advantages described above, we employ effective-medium approximation [26,27] and convert the corrugated rod into an equivalent multilayered homogenized one [18]. In this way, one can directly control the external electromagnetic characteristics of the wires by adjusting the volume ratio of the two materials. In order to acquire a strong transverse polarizability for the homogenized particles [28,29], we select that ratio accordingly. To this end, we consider multiple cylinders, parallel to each other, in a metasurface layout which is illuminated by an obliquely propagated plane wave. Under the condition of electrically small cylinders, we perform a dipole approximation of their response; such an assumption does not affect the developed far field [15]. After rigorously summing [30] that scattering field using every one of the rods, we obtain explicit forms for the reflected and transmitted beams of various refraction orders [31] produced by the grating. In this sense, we can find design parameters for this summation that lead to lattice resonances, namely, constructive or destructive interference from the response of the grating's cylinders [19].

With realistic choices of sizes and permittivities, we notice that line-of-sight transmissivity, namely, the power carried by the transmitted beam following Snell's law, changes abruptly when new refraction orders are about to emerge, regardless of the input polarization. This sharp variation in the response of the device renders it suitable to use as a photonic switch or highly selective filter. We also examine the patterns of the power channeled along unconventional directions when anomalous reflection/transmission occurs, and we represent the vectorial energy equilibrium in characteristic cases. Finally, we regard actual lossy media in the visible, and we report almost perfect absorption for specific spatial distributions of the particles, which are practically independent of their own size. Such parametric sensitivity of absorption of our lattice can be directly exploited in sensing and radar invisibility systems.

\section{MATHEMATICAL FORMULATION AND MODELING}

\section{A. Single cylindrical particle}

The building block of our device is depicted in Fig. 1(a), where the Cartesian $(x, y, z)$ and cylindrical $(r, \varphi, z)$ coordinate systems are also defined. That cylindrical particle has infinite length and a circular cross section of radius $a$ and is filled with a material with complex relative permittivity $\varepsilon_{\text {meta }}$. Its volume has been linearly corrugated with period $h$, so that rotationally symmetric wedges of width $(a-g)$ and height $(h-d)$ are formed, which are filled with another material of permittivity $\varepsilon_{\text {diel }}$. The time dependence is considered to be periodic $e^{+j k_{0} c t}$ and is suppressed throughout the analysis, where $c$ is the speed of light and $k_{0}=2 \pi / \lambda_{0}$ is the operating wave number, all in vacuum.

The corrugation period $h$ along axis $z$ of our cylinder is taken to be much smaller than the oscillating free-space wavelength $\lambda_{0}$; such a feature enables us to convert the structure into an analytically solvable equivalent one, which remains unaltered along the $z$ axis. If, additionally, the excitation is $z$
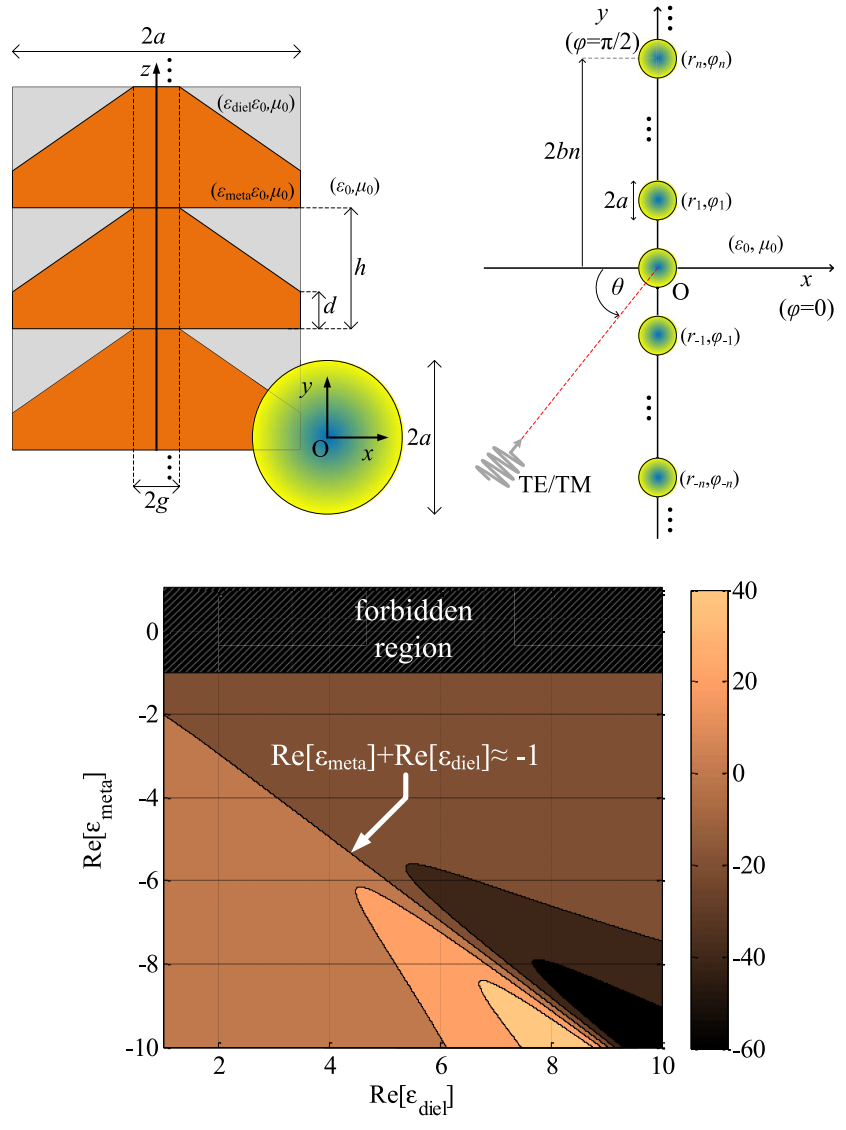

(c)

FIG. 1. (a) Side view of a periodically corrugated cylindrical particle composed of two media with permittivities $\varepsilon_{\text {meta }}$ and $\varepsilon_{\text {diel }}$. The top view of the homogenized analog is given in the bottom right inset. (b) Top view of the periodical array of the homogenized particles in (a) excited by an oblique TE/TM plane wave with incidence angle $\theta$. (c) Variation of the effective permittivity $\operatorname{Re}\left[\varepsilon_{1}^{\|}\right]$of a homogenized single particle's external layer on the $\left(\operatorname{Re}\left[\varepsilon_{\text {diel }}\right], \operatorname{Re}\left[\varepsilon_{\text {meta }}\right]\right)$ map while keeping $\operatorname{Re}\left[\varepsilon_{1}^{\perp}\right]=-1$.

independent, we have the simplified decoupling of the fields in the TE set (magnetic field parallel to the $z$ axis, electric field on the $x y$ plane) and the TM set (electric field parallel to the $z$ axis, magnetic field on the $x y$ plane). In particular, the wave interaction of the corrugated cylinder would be almost identical to that of a two-dimensional (2D) multilayered one with $U$ concentric regions based on effective-medium approximation [26,27] for the hollow cylindrical volumes defined by $(U-u) / U<r / a<(U-u+1) / U$, with $u=$ $1, \ldots, U$. The index $u$ increases from the external surface $(u=1)$ to the core $(u=U)$, and each of the concentric layers has the same thickness $a / U$. The effective permittivities of the layer with index $u$ are given by the well-known formulas [18] $\varepsilon_{u}^{\perp}=\varepsilon_{\text {meta }} d_{u} / h+\varepsilon_{\text {diel }}\left(1-d_{u} / h\right)$ (for the $x y$ plane, TE polarization) and $\frac{1}{\varepsilon_{u}^{\| 1}}=\frac{d_{u} / h}{\varepsilon_{\text {meta }}}+\frac{1-d_{u} / h}{\varepsilon_{\text {diel }}}$ (for the $z$ axis, TM polarization), provided that $h \ll \lambda_{0}$ for homogenization purposes. The auxiliary quantities $d_{u}$ and $\rho_{u}$ (outer surface radius) for the $u$ th cladding $(u=1, \ldots, U)$ of the multilayered analogs 
are

$$
\frac{d_{u}}{h}=\frac{d}{h}+\left(1-\frac{d}{h}\right) \frac{a-\rho_{u}}{a-g}, \quad \frac{\rho_{u}}{a}=\frac{U-u+1}{U} .
$$

After the homogenization process described above, the field in the multilayered equivalent cylinder would have the form of infinite sums of the Helmholtz canonical solutions in the cylindrical coordinate system. Given the fact that the rod in Fig. 1(a) would serve the role of a structural unit for a more complex device, it is sensible to take it to be electrically small, namely, with a radius $a$ much smaller than the vacuum wavelength $\lambda_{0}$. Accordingly, we are permitted to describe the field's spatial variation across its volume by keeping only the first two azimuthal orders [15] of the aforementioned solution series (omnidirectional and dipole terms). Under this approximation and regardless of the source feeding such a particle, the $z$-directed field $\mathbf{G}_{\text {ext }}=\hat{\mathbf{z}} G_{\text {ext }}$ (magnetic for TE polarization and electric for TM polarization) in the external layer $u=1$ will be written as

$$
\begin{aligned}
G_{\mathrm{ext}}(r, \varphi)= & C_{0}\left[J_{0}\left(k_{1} r\right)+F_{0} H_{0}^{(2)}\left(k_{1} r\right)\right] \\
& +C_{1 c}\left[J_{1}\left(k_{1} r\right)+F_{1 c} H_{1}^{(2)}\left(k_{1} r\right)\right] \cos \varphi \\
& +C_{1 s}\left[J_{1}\left(k_{1} r\right)+F_{1 s} H_{1}^{(2)}\left(k_{1} r\right)\right] \sin \varphi .
\end{aligned}
$$

The symbols $J_{p}, H_{p}^{(2)}$ are used for Bessel and (second-type) Hankel functions of $p$ th order, and $k_{1}=k_{0} \sqrt{\varepsilon_{1}}$ is the wave number corresponding to the material of the external layer of our particle $\left(\varepsilon_{1}=\varepsilon_{1}^{\perp}\right.$ for TE polarization and $\varepsilon_{1}=\varepsilon_{1}^{\|}$ for TM polarization). The complex constants $\left(C_{0}, C_{1 c}, C_{1 s}\right)$ are arbitrary and are determined by the illuminating source creating the background field.

In contrast, the proportionality constants $\left(F_{0}, F_{1 c}, F_{1 s}\right)$ are specific and characteristic of the homogenized wire since they reveal the analogy between $r$-dependent solutions $\left(J_{p}, H_{p}^{(2)}\right)$ within the external $(u=1)$ layer. It is evident that $F_{0}=F_{1 c}=F_{1 s}=0$ if the cylinder is a homogeneous rod. These constants are found by imposing the boundary conditions at the internal interfaces $r=\rho_{u}$ for $u=2, \ldots, U$ of the multilayered cylinder. More specifically, we obtain $F_{0}=\left[\prod_{u=2}^{U} \mathbf{T}_{u}(0)\right]_{21} /\left[\prod_{u=2}^{U} \mathbf{T}_{u}(0)\right]_{11}$ and $F_{1 c}=$ $F_{1 s}=\left[\prod_{u=2}^{U} \mathbf{T}_{u}(1)\right]_{21} /\left[\prod_{u=2}^{U} \mathbf{T}_{u}(1)\right]_{11}$, where $\mathbf{T}_{u}(p)$ is the $2 \times 2$ transfer matrix [32] from the $u$ th layer to the $(u-1)$ th layer (from the core to the external surface $r=a$ ) for the assumed polarization (TE/TM), while $p$ is the corresponding azimuthal order [15]. The explicit expressions of the matrices $\mathbf{T}_{u}^{T E}(p)$ and $\mathbf{T}_{u}^{T M}(p)$ are omitted for brevity.

\section{B. Infinite metasurface}

When one locates infinitely many of these particles parallel to the $z$ axis with a constant distance $2 b$ between two neighboring ones along the $y$ axis, one makes the metasurface in Fig. 1(b). The response from every single cylinder can be easily expressed in the corresponding local coordinate system $\left(r_{n}, \varphi_{n}, z\right)$ centralized at the $O_{n}$ axis of each $\operatorname{rod}[n \in \mathbb{Z}$, as shown in Fig. 1(b)]. Given the fact that such a grating is illuminated by an obliquely incident plane wave of axial (z-polarized) field (magnetic for the TE case and electric for the TM case) written as [33] $G_{\text {inc }}=$ $e^{-j k_{0}(x \cos \theta+y \sin \theta)}=\sum_{p=-\infty}^{+\infty} j^{-p} J_{p}\left(k_{0} r\right) e^{-j p(\varphi-\theta)}$, the scattering field of the whole metasurface readily takes the form

$$
\begin{aligned}
G_{\text {scat }}= & \sum_{n=-\infty}^{+\infty} e^{-2 j k_{0} b n \sin \theta}\left[S_{0} H_{0}^{(2)}\left(k_{0} r_{n}\right)\right. \\
& \left.+S_{1 c} H_{1}^{(2)}\left(k_{0} r_{n}\right) \cos \varphi_{n}+S_{1 s} H_{1}^{(2)}\left(k_{0} r_{n}\right) \sin \varphi_{n}\right] .
\end{aligned}
$$

We note that (3) is a function of all the local cylindrical coordinates $\left(r_{n}, \varphi_{n}, n \in \mathbb{Z}\right)$ and written under the assumption of dipole approximation. Indeed, due the infinite size of the structure and the infinite wave front of the excitation, the scattering from the arbitrary $n$th particle will be spatially identical to that of any other particle with a phase difference $e^{-2 j k_{0} b n \sin \theta}$ dictated by its position $O_{n}$ around axis $(x, y)=$ $(0,2 b n)$. Therefore, the coefficients $\left(S_{0}, S_{1 c}, S_{1 s}\right)$ are not functions of $n$ and describe the interaction of the central $(n=0)$ wire with the impressed field.

By employing the addition theorem for circular cylindrical waves [30], we can find a formula for the scattering field expressed in the global cylindrical coordinate system $(r, \varphi, z)$, associated with the central particle:

$$
\begin{aligned}
G_{\text {scat }}(r, \varphi)= & \left(S_{0} \Sigma_{0}-S_{1 s} \Sigma_{1}\right) J_{0}\left(k_{0} r\right)+S_{0} H_{0}^{(2)}\left(k_{0} r\right) \\
& +S_{1 c}\left[\left(\Sigma_{0}+\Sigma_{2}\right) J_{1}\left(k_{0} r\right)+H_{1}^{(2)}\left(k_{0} r\right)\right] \cos \varphi \\
& +\left\{\begin{array}{c}
\left.2 S_{0} \Sigma_{1}+S_{1 s}\left(\Sigma_{0}-\Sigma_{2}\right)\right] J_{1}\left(k_{0} r\right) \\
+S_{1 s} H_{1}^{(2)}\left(k_{0} r\right)
\end{array}\right\} \sin \varphi .
\end{aligned}
$$

The symbols $\Sigma_{0}, \Sigma_{1}, \Sigma_{2}$ are used for infinite sums involving Hankel functions of the respective order. The derivation of (4) and the numerical evaluation of these series are described in the Appendix. To this end, it is sufficient to impose the necessary boundary conditions on the $n=0$ cylinder at $r=a$ since the rest of the particles interact trivially differently from the central one (due to the aforementioned phase difference). Indeed, we have only six complex unknowns $\left(C_{0}, C_{1 c}, C_{1 s}, S_{0}, S_{1 c}, S_{1 s}\right)$ and two boundary conditions at $r=a$ : (i) the Dirichlet condition, $G_{\text {inc }}+G_{\text {scat }}=G_{\text {ext }}$, and (ii) the Neumann condition, $\frac{\partial G_{\text {inc }}}{\partial r}+\frac{\partial G_{\text {scat }}}{\partial r}=\frac{1}{\varepsilon_{1}^{\perp}} \frac{\partial G_{\text {ext }}}{\partial r}$ for TE polarization and $\frac{\partial G_{\text {inc }}}{\partial r}+\frac{\partial G_{\text {scat }}}{\partial r}=\frac{\partial G_{\text {ext }}}{\partial r}$ for TM polarization. Note that all the unknowns are uniquely determined since each boundary condition will contribute three scalar equations (one omni-directional, another proportional to $\cos \varphi$, and the last proportional to $\sin \varphi$ ). It should be stressed that the dipole approximation is also performed in the incident wave since a small particle cannot "feel" a substantial azimuthal change of the background field.

After finding the coefficients $S_{0}, S_{1 c}, S_{1 s}$, the scattering field can be written in the global Cartesian coordinate system by employing the Poisson summation formula [34]:

$$
\begin{gathered}
G_{\text {scat }}(x, y)=e^{-j k_{0} y \sin \theta} \sum_{m=-\infty}^{+\infty} e^{-j m \pi y / b} \frac{e^{-\kappa_{m}|x| / b}}{\kappa_{m}} \\
\times\left[j S_{0}+\frac{j \kappa_{m} S_{1 c} \operatorname{sgn}(x)-S_{1 s}\left(m \pi+k_{0} b \sin \theta\right)}{k_{0} b}\right],
\end{gathered}
$$


where $\kappa_{m}=\sqrt{\left(m \pi+k_{0} b \sin \theta\right)^{2}-\left(k_{0} b\right)^{2}}$. The derivation of (5) is described in the Appendix.

If we are interested in the electromagnetic response far from the structure $(x \rightarrow \pm \infty)$, we will take into account only the refraction orders $m \in \mathbb{Z}$ belonging to the set $\mathcal{M}$ defined by the double inequality $[14,19]$ :

$$
-\left\lfloor\frac{2 b}{\lambda_{0}}(1+\sin \theta)\right\rfloor \leqslant m \leqslant\left\lfloor\frac{2 b}{\lambda_{0}}(1-\sin \theta)\right\rfloor,
$$

for which $\kappa_{m}$ is purely imaginary. Obviously, if $\frac{2 b}{\lambda_{0}}<\frac{1}{1+|\sin \theta|}$, only Snell's transmission and reflection are supported by our metasurface. The scattered far field of the grating towards the right $(x>0)$ and left $(x<0)$ sides can be evaluated directly from (5). To the (always present) ordinary $(m=$ 0 ) transmitted order, one should add [15] the background incident field $G_{\text {inc }}(x, y)$. In this sense, the transmission $T_{m}$ and reflection $R_{m}$ coefficients are determined; thus, the transmitted $z$-polarized field (TE/TM) is written as $G_{\text {tran }}(x, y)=$ $\sum_{m \in \mathcal{M}} T_{m} e^{-j k_{0}\left(x \cos \theta_{m}+y \sin \theta_{m}\right)}$, while the reflected one reads $G_{\text {ref }}(x, y)=\sum_{m \in \mathcal{M}} R_{m} e^{-j k_{0}\left(-x \cos \theta_{m}+y \sin \theta_{m}\right)}$. The common angle of propagation (measured from the horizontal $x$ axis) of the transmitted (traveling towards $x>0$ ) and the reflected (towards $x<0$ ) waves of order $m$ is given by

$$
\theta_{m}=\arcsin \left(\sin \theta+\frac{m \pi}{k_{0} b}\right) .
$$

If $\theta_{m}<0$ when $\theta>0$ for a specific $m \in \mathcal{M}$, that order of refraction is called anomalous; namely, the developed transmitted/reflected rays propagate towards the same region of space in which the metasurface is excited $(y<0$ half space). For $m=0$, (7) yields a trivial result: $\theta_{0}=\theta$ (Snell's transmission/reflection).

\section{Transverse external permittivity}

Returning to the single-particle configuration in Fig. 1(a), the periodical corrugations along its axis offer significant design advantages since numerous alternative structures can be obtained with the sole use of two media [25]: $\left(\varepsilon_{\text {meta }}, \varepsilon_{\text {diel }}\right)$. Indeed, by choosing the two permittivity functions to be as distinct as possible, namely, the first one having $\operatorname{Re}\left[\varepsilon_{\text {meta }}\right]<1$ (the subscript stands for either metal or metamaterial) and the second one having $\operatorname{Re}\left[\varepsilon_{\text {diel }}\right]>1$ (the subscript stands for common dielectric), a number of interesting and diverse responses can be recorded. The structure of Fig. 1(a) possesses two additional merits: (i) The designer can engineer the external (or any other) equivalent permittivity by varying the inclination of corrugations. (ii) The linear change in the effective permittivity makes the material transition smooth and encourages interplay between fields within the effective cylindrical multilayers and the incident illumination.

In this work, we choose to give the effective permittivity of the external layer $\varepsilon_{1}^{\perp}$ (for TE waves), a specific value dictated by the dipole moment of an elongated cylindrical wire. In particular, it is well known that for the case of a homogeneous infinite rod [28,29], the transverse polarizability behaves as $\alpha_{\perp} \sim \frac{\varepsilon^{\perp}-1}{\varepsilon^{\perp}+1}$, while the longitudinal one is just proportional to the permittivity contrast: $\alpha_{\|} \sim\left(\varepsilon^{\|}-1\right)$. That singularity (maximization in practice) in $\alpha_{\perp}$ means that a choice $\operatorname{Re}\left[\varepsilon_{1}^{\perp}\right]=-1$ will make the cylinder interact strongly with the excitation through its outer surface. Such a resonance happens due to the metallic nature of this surface and is commonly called a localized surface plasmon $[35,36]$. The permittivity for which this effect occurs, changes with respect to the particle's shape; for example, spheres made of metals or metal-dielectric mixtures have plasmonic resonance at the operational frequency where $\varepsilon=-2$.

A typical variation of the (real part of) external equivalent permittivity $\varepsilon_{1}^{\|}$for the other (TM) polarization with respect to the real dielectric constants of the two media while the TE resonant condition holds, is shown in Fig. 1(c). First of all, the equality $\operatorname{Re}\left[\varepsilon_{1}^{\perp}\right]=-1$ is possible only when $\operatorname{Re}\left[\varepsilon_{\text {meta }}\right]<-1$; the infeasible material combinations are labeled the "forbidden region." In addition, a large change in the value of $\operatorname{Re}\left[\varepsilon_{1}^{\|}\right]$ is recorded in the vicinity of the line $\operatorname{Re}\left[\varepsilon_{\text {meta }}\right]+\operatorname{Re}\left[\varepsilon_{\text {diel }}\right]=$ -1 , which becomes more rapid for more negative $\operatorname{Re}\left[\varepsilon_{\text {meta }}\right]$ and less lossy designs (unbounded $\varepsilon_{1}^{\|}$in the lossless scenario). From Fig. 1(c), it is clear that while keeping $\operatorname{Re}\left[\varepsilon_{1}^{\perp}\right]=-1$, the equivalent external permittivity $\operatorname{Re}\left[\varepsilon_{1}^{\|}\right]$can cover a wide range of values, and thus, we expect the responses for the two polarizations to be quite different.

\section{NUMERICAL RESULTS AND DISCUSSION}

\section{A. Input parameters and output quantities}

In the following numerical examples, we always obey the condition $\operatorname{Re}\left[\varepsilon_{1}^{\perp}\right]=-1$. Furthermore, we avoid changing the properties of the two media in order to understand what utilities are possible for a specific set of permittivities. Therefore, we keep them constant and equal to values that can be found easily at optical frequencies: $\varepsilon_{\text {meta }} \cong-2$ and $\varepsilon_{\text {diel }} \cong 3$. The electrical size of the cylinders $a / \lambda_{0}$ is kept moderate since they are playing the role of particles in the metasurface. The radius $g$ of the core filled with $\varepsilon_{\text {meta }}$ has been selected to be much smaller than the radius $a$ of the particles in order to guarantee smoother texture transitions $(g=0.2 a)$. When it comes to the number of equivalent layers $U$, it is chosen to be sufficiently large for the results to converge.

As far as the power of each of the refracted waves is concerned, it is meaningful only along the normal-to-metasurface $x$ direction since the incident field dictates the $y$ variation and the corresponding power flow. For this reason, we define the power carried by the transmitted and reflected modes of order $m$ (as a fraction of the unitary incident power) through the following respective normalized quantities:

$$
P_{m}^{\text {tran }}=\left|T_{m}\right|^{2} \frac{\cos \theta_{m}}{\cos \theta}, \quad P_{m}^{\mathrm{ref}}=\left|R_{m}\right|^{2} \frac{\cos \theta_{m}}{\cos \theta},
$$

which are the basic observables of our study. Since we have multiple directions of propagating waves, the energy conservation is written in the form $\sum_{m \in \mathcal{M}}\left(P_{m}^{\text {tran }}+P_{m}^{\text {ref }}\right)=1$, expressing the power exchange only along the $x$ axis [14,31].

One of the major approximations of the present work is that we keep only the first two terms of the azimuthal variation in the corresponding canonical field expansions. Such a reduction, surprisingly, affects only the near-field distribution around and into the cylinders instead of the metasurface behavior in the far region (transmitted and reflected rays). 


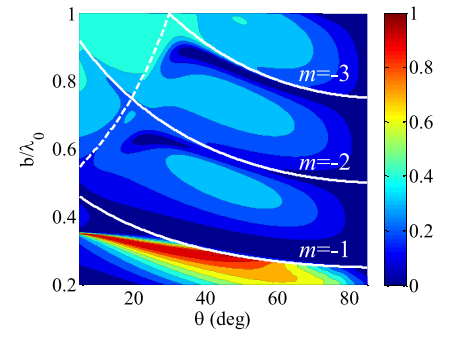

(a)

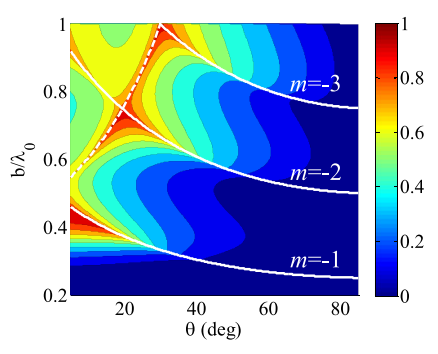

(b)
FIG. 2. The power carried by Snell's transmission $P_{0}^{\text {tran }}=\left|T_{0}\right|^{2}$ in a contour plot with respect to the angle of incidence $\theta$ and the half period of the metasurface divided by the free-space wavelength $b / \lambda_{0}$ for (a) TE polarization and (b) TM polarization. Plot parameters: $\varepsilon_{\text {meta }}=-2, \varepsilon_{\text {diel }}=3, a=0.2 \lambda_{0}, g=0.2 a$.

Indeed, due to periodicity, highly oscillating azimuthal harmonics $e^{j p \varphi}$ for $p>1$ have a null average contribution in the far-field response of the entire device [15]. In our numerical examples, we verify energy conservation up to the third decimal digit for every single case of lossless design. This is a strong indication that neither the bipolar approximation adopted in (2)-(5) nor the homogenization described by (1) affects the validity of the far-field results. When losses are considered, our observable is no longer the power carried by the modes but the electromagnetic absorption $A=1-$ $\sum_{m \in \mathcal{M}}\left(P_{m}^{\text {tran }}+P_{m}^{\text {ref }}\right)$ for each polarization; such a normalized quantity gives the percentage of the unitary input power which gets absorbed within the grating. It should be mentioned that in the following numerical results all the represented quantities concern the far region and thus do not contain approximation errors (unlike the near fields, which are not computed or shown). Even absorption $A$, which takes place in the volume of the particles, is defined in terms of far-field transmitted $P_{m}^{\text {tran }}$ and reflected $P_{m}^{\text {ref }}$ power.

As far as the fabrication of the described metasurface structure is concerned, one can mimic the technique employed in a similar setup [18]. Suitably corrugated metallic wires can be embedded in transparent dielectrics and can be placed next to each other with help from a three-dimensionally printed plastic array by using stereolithography. Furthermore, since the characteristics of the equivalent concentric particles have been obtained (Sec. II A), construction methods of $z$-invariant parallel rods [37] are suitable; alternatively, emulating effective corrugations around the metallic cores through conformal films [38], is also possible.

\section{B. Line-of-sight transmission}

Let us consider a metasurface composed of particles with moderate but non-negligible size $\left(a=0.2 \lambda_{0}\right)$ and the specifications mentioned in Sec. III A. In this section, our aim is to investigate the response variation for most metasurfaces comprising such cylinders at a fixed operating wavelength $\lambda_{0}$. In Fig. 2, we show the line-of-sight transmitted power, namely, the power $P_{0}^{\operatorname{tran}}=\left|T_{0}\right|^{2}$ carried by Snell's $(m=0)$ transmission, in a contour plot as a function of the incidence angle $\theta$ and the grating's electrical half period $b / \lambda_{0}$ for both polarizations. Combinations of $\theta$ and $b / \lambda_{0}$ which, according to the equalities of (6), signify the appearance of a new

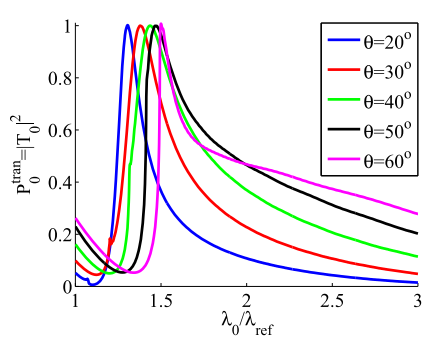

(a)

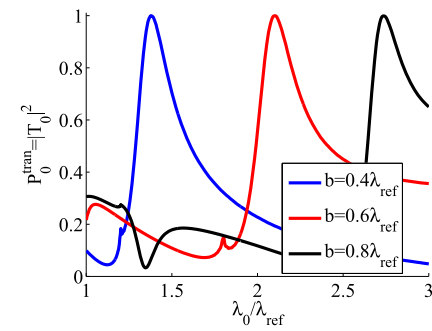

(b)
FIG. 3. The power carried by Snell's transmission $P_{0}^{\text {tran }}=\left|T_{0}\right|^{2}$ for TE polarization of Fig. 2(a) as a function of operating wavelength $\lambda_{0} / \lambda_{\text {ref }}$ for (a) various incidence angles $\theta\left(a=0.2 \lambda_{\text {ref }}=0.5 b\right)$ and (b) various metasurface spatial half periods $b / \lambda_{\text {ref }}\left(a=0.2 \lambda_{\text {ref }}, \theta=\right.$ $30^{\circ}$ ).

refraction order $m$ are shown in Fig. 2 by white lines (solid for negative orders, dashed for the only positive one: $m=1$ ). The monotonicity of these loci on the $\left(\theta, b / \lambda_{0}\right)$ map is determined by their signs, which means that lines of opposite-sign $m$ can cross each other, indicating the simultaneous emergence of two additional refraction orders.

In Fig. 2(a), where TE excitation is considered, we notice that $P_{0}^{\text {tran }}$ gets sharply maximized (by taking almost $100 \%$ values) close to the boundary of the $m=-1$ order; once the metasurface becomes sparse enough to support anomalous refraction $(m=-1), P_{0}^{\text {tran }}$ vanishes. Indeed, once a new refraction order gets triggered, it directly absorbs a lot of energy previously carried by Snell's transmission. We also note that line-of-sight transmissivity exhibits secondary maxima in between two loci of consecutive anomalous orders. Furthermore, the critical $b / \lambda_{0}$ for which maximum $P_{0}^{\text {tran }}$ is observed, is not very sensitive to incidence angle changes, giving an almost horizontal, thin, and lengthy "plateau" of large $\left|T_{0}\right|^{2}$ values in the map in Fig. 2(a). In Fig. 2(b), where TM waves are examined, $P_{0}^{\text {tran }}$ is enhanced (with magnitudes close to $100 \%$ of the unitary incident power) at the intersection points of two loci corresponding to opposite-sign $m$. Similar to the TE case, the power carried by the line-of-sight transmission feeds the two pairs of freshly developed refractive waves.

In Fig. 3, we examine the TE case of Fig. 2(a) and represent the line-of-sight transmission as a function of the operational wavelength $\lambda_{0} / \lambda_{\text {ref }}$ ( $\lambda_{\text {ref }}$ is a constant reference wavelength dictated by the application). In Fig. 3(a), we consider various incidence angles $\theta$ and observe multiple peaks within a short range of operating frequencies [demonstrating the $\theta$ insensitivity mentioned in Fig. 2(a)]. The most important feature is that a small shift in $\lambda_{0}$ can lead to a dramatic increase of $\left|T_{0}\right|^{2}$ (from almost $0 \%$ to $100 \%$ ), revealing extremely high selectivity in the frequency response of our device. In Fig. 3(b), we are referring again to the plateau in Fig. 2(a) and considering several distances $b$. As in Fig. 3(a), we observe sharp maxima in $P_{0}^{\text {tran }}$ but at very different wavelengths $\lambda_{0}$, which means that, by tuning $b$, one can reach maximal lineof-sight transmissivity for the desired frequencies. This interesting frequency-selective behavior gives evidence that our metasurface can be used for switching and filtering purposes.

In Fig. 4, we perform the same considerations as in Fig. 3, with the same $\lambda_{\text {ref }}$, but for the TM waves [of Fig. 2(b)]. In Fig. 4(a), we notice that the influence of the angle $\theta$ is similar 


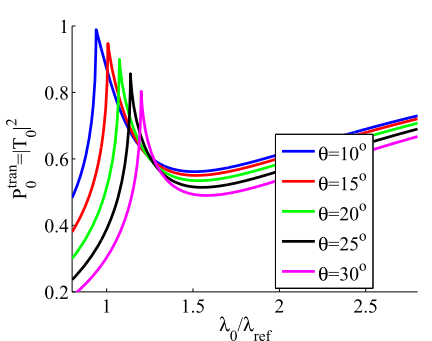

(a)

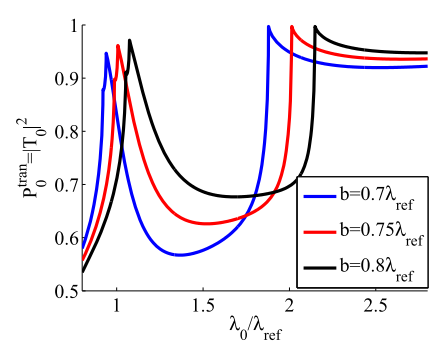

(b)
FIG. 4. The same as Fig. 3 but for TM polarization of Fig. 2(b) as a function of $\lambda_{0} / \lambda_{\text {ref }}$ for (a) various $\theta\left(a=0.2 \lambda_{\text {ref }}=0.5 b\right)$ and (b) various $b / \lambda_{\text {ref }}\left(a=0.2 \lambda_{\text {ref }}, \theta=20^{\circ}\right)$.

to that of Fig. 3(a), enabling the grating to act as a selector of operational frequency. However, the maxima appear at a different band [note that the metasurfaces of Figs. 3(a) and 4(a) are identical] and are weaker, once our incident plane wave gets more oblique. For a specific wavelength $\lambda_{0}$, the dissimilar way that the device treats TE and TM waves renders it suitable as a polarization separator. In Fig. 4(b), where various spatial periods are considered, we observe two peaks of $\left|T_{0}\right|^{2}$ [corresponding to the local maxima of Fig. 2(b)] instead of the single one in Fig. 3(b) for the same size, $2 \lambda_{\text {ref }}$, of the wavelength band. It should finally be stressed that in the TM polarization the peaks look locally sharper, but the minimum-to-maximum range of the curves is much shorter than the corresponding one in Fig. 3 (TE polarization).

\section{Anomalous refraction}

In contrast to planar homogeneous layers, excitation of periodic gratings is usually accompanied by development of waves traveling in directions not predicted by Snell's law. This anomalous refraction is the major factor affecting the lineof-sight transmission apart from ordinary $(m=0)$ reflection $\left|R_{0}\right|^{2}$. Therefore, it is worth examining the variation of power $P_{m}^{\text {tran/ref }}$ by fields of anomalous refraction order $m$. Let us confine our research to TE polarization (in particular, the cases considered in Fig. 3), where particles are expected to respond more vividly (since $\varepsilon^{\perp}=-1$ ).

In Fig. 5(a), we represent the power carried by the developed transmitted (solid lines) and reflected (dashed lines) waves as functions of $\lambda_{\text {ref }} / \lambda_{0}$, which is proportional to the operational frequency [Fig. 3(a) for $\theta=40^{\circ}$ ]. It is easy to observe the frequencies beyond which each refraction order is activated, and in most cases, the corresponding power takes substantial (or even maximal) values. This feature also affects the behavior of already developed waves according to the inevitable energy equilibrium. For example, one can observe the sharp peak of $m=-1$ anomalous transmission once $m=-2$ refraction emerges, at the expense of Snell's (line-of-sight) transmission. Three distinct frequencies with interesting power distributions are indicated with markers of different colors and placed on the corresponding curves. In Fig. 5(b), we schematically show with arrows the directions and the squared magnitudes of propagating waves for these three characteristic frequencies using the respective colors. Note that conservation of energy may be visually violated since $\left|T_{m}\right|^{2},\left|R_{m}\right|^{2}$, according to (8), can be smaller or larger

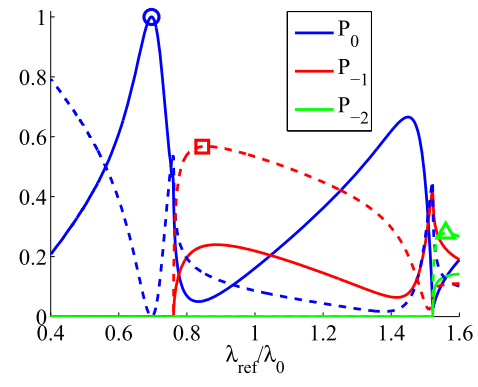

(a)

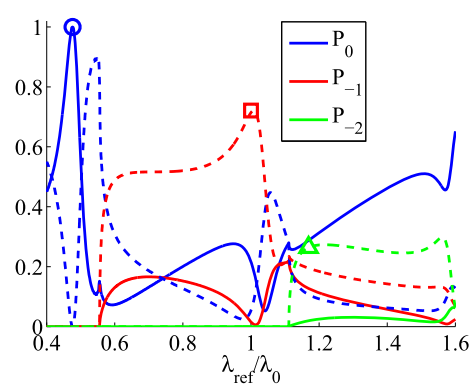

(c)

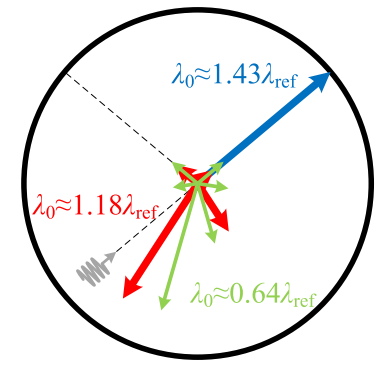

(b)

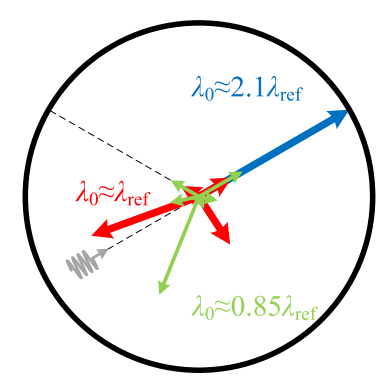

(d)
FIG. 5. (a) The power $P_{m}^{\text {tran/ref }}$ carried by various transmission (solid lines) and reflection (dashed lines) orders $m$ as a function of the operational frequency $\lambda_{\text {ref }} / \lambda_{0}$ for the case of Fig. 3(a) with $\theta=40^{\circ}$. (b) Schematic of the squared magnitudes and directions of waves for the wavelengths marked in (a). (c) The same as (a), but for the case of Fig. 3(b) with $b=0.6 \lambda_{0}$. (d) The same as (b), but for the wavelengths marked in (c).

than $P_{m}^{\text {tran }}, P_{m}^{\text {ref }}$. The incidence direction and ordinary reflection angles are represented by black dashed lines. We directly notice almost $100 \%$ line-of-sight transmissivity at $\lambda_{0} \cong 1.43 \lambda_{\text {ref }}$, as shown in Fig. 3(a). Furthermore, we note the anomalous reflection at $\lambda_{0} \cong 1.18 \lambda_{\text {ref }}$, while a typical scattering of power along six different directions is recorded at $\lambda_{0} \cong 0.64 \lambda_{\text {ref }}$. In Fig. $5(\mathrm{c})$, we repeat our computations for the metasurface in Fig. 3(b) with $b=0.6 \lambda_{\text {ref }}$. The substantial anomalous reflection sending a major part of the power back to the source at $\lambda_{0} \cong \lambda_{\text {ref }}$ deserves special mention. Again, three characteristic cases are marked, and the corresponding propagation directions are presented in Fig. 5(d).

\section{Absorption}

In all the previous examples, we considered lossless media $\left(\operatorname{Im}\left[\varepsilon_{\text {meta }}\right]=\operatorname{Im}\left[\varepsilon_{\text {diel }}\right]=0\right)$. When losses are present (which is inevitable, especially for $\varepsilon_{\text {meta }}$ ), our metasurface absorbs part of the incoming energy, a feature not always unwanted. Indeed, thin planar absorbers [39] are extremely useful components in a large number of electromagnetic devices, including switching absorbers/reflectors [40], sensors plates, and polarization tuning gratings. In this section, we will present maximally absorbing designs of Fig. 1 by employing actual lossy media. In other words, we do not optimize the losses of the materials, that is, search for maximum absorption $A$ between two extrema of zero and infinite losses (giving $A=$ 0 ). We instead adjust our structure to already existing and realistic textures, so that very high absorbance is achieved. 


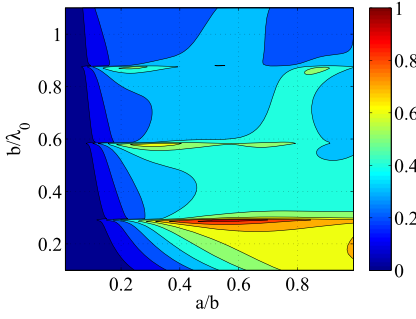

(a)

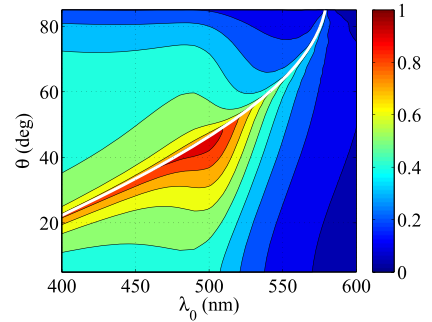

(b)

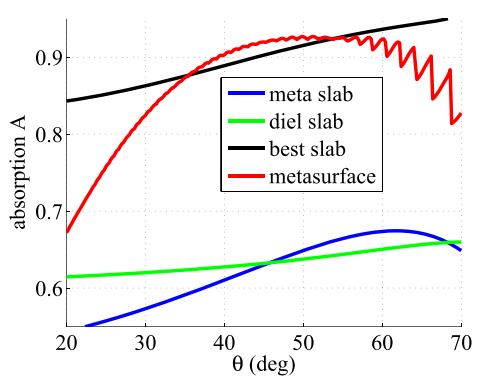

(c)

FIG. 6. (a) Absorbed power $A$ in a contour plot with respect to the distance ratios $a / b$ and $b / \lambda_{0}\left(\lambda_{0}=500 \mathrm{~nm}, \theta=45^{\circ}\right)$. (b) Absorbed power $A$ in contour plot with respect to wavelength $\lambda_{0}$ and incidence $\theta$. (c) Absorbed power $A$ as a function of $\theta$ $\left(a \cong 0.15 \lambda_{0}\right.$ ) for various homogeneous slab configurations and our metasurface. Particles are made of gold and lossy aluminum oxide.

Let us consider two media: gold for $\varepsilon_{\text {meta }}$ and aluminum oxide with fixed engineered losses $\left(\operatorname{Im}\left[\varepsilon_{\text {diel }}\right]=-0.9\right)$ for $\varepsilon_{\text {diel }}$ employed around $\lambda_{0} \cong 500 \mathrm{~nm}$ (green light). The ratio $d / h$ from Fig. 1(a) is chosen so that $\operatorname{Re}\left[\varepsilon_{1}^{\perp}\right]=-1$, as described in Sec. II C, and TE waves are considered (as in Fig. 5).

In Fig. 6(a), we represent the absorption $A$ in a contour plot as a function of the normalized radius of the particles $a / b$ and the electrical half period $b / \lambda_{0}$ of the metasurface. We directly observe a series of sharp maxima (reaching 100\%) with respect to $b / \lambda_{0}$, occurring all at the same $b \cong \frac{\lambda_{0}}{2+\sqrt{2}}$, which, according to (6), corresponds to the appearance of the $m=$ -1 refraction order (for $\theta=45^{\circ}$ ). The same region of the map $\left(a / b, b / \lambda_{0}\right)$ gives maximum line-of-sight transmission $P_{0}^{\text {tran }}=\left|T_{0}\right|^{2}$ for the analogous lossless designs $\left(\operatorname{Im}\left[\varepsilon_{\text {meta }}\right]=\right.$ $\operatorname{Im}\left[\varepsilon_{\text {diel }}\right]=0$ ). Weaker local maxima are noted at distances $b$, triggering a new refraction order. It is also remarked that similar behavior is exhibited (obviously at different $b$ ) for alternative choices of the incidence angle $\theta$.

In Fig. 6(b), we consider a structure corresponding to a point on the distance map $\left(a / b, b / \lambda_{0}\right)$ in Fig. 6(a) which exhibits substantial absorption of incoming power. We test it for various incidence angles $\theta$ of TE fields and operational wavelengths $\lambda_{0}$ by taking into account the material dispersion [41]. We observe that the maximum absorption pattern coincides with the locus associated with the $m=-1$ refraction order (indicated by a solid while line), in accordance with the conclusions of Fig. 6(a) and the variations of Fig. 2. Once we change $\lambda_{0}$ or $\theta$ to excite our metasurface with waves different from them dictated by the aforementioned line, we experience a drop in the absorption which is naturally more rapid once we are far from the selected operation point $\left(\lambda_{0}=500 \mathrm{~nm}\right.$, $\left.\theta=45^{\circ}\right)$.
The advantage of our structure in terms of TE absorption is finally demonstrated in Fig. 6(c), where absorbance $A$ of the metasurface of Fig. 1(b) is compared with that of various uniform slabs with the same size $2 a$ for all incidence angles. We use the same media (gold and lossy aluminum oxide) operated at the same wavelength $\lambda_{0}=500 \mathrm{~nm}$ as in Fig. 6(a), while the cylinder radius takes a rather typical value: $a \cong 0.15 \lambda_{0}$. For each angle $\theta$ we optimized the distance $b$ defining our grating in terms of maximum $A$. Naturally, homogeneous layers of thickness $2 a$ filled with the constituent materials of our particles do a much worse job for all directions of illumination. What is, however, remarkable is that our metasurface using the same particles and simply rearranging them accordingly can beat the highest absorbing uniform layer (fully optimized with respect to $(\operatorname{Re}[\varepsilon], \operatorname{Im}[\varepsilon])$ at each $\theta$ ) for a wide range of incidence directions: $35^{\circ}<\theta<55^{\circ}$. Note that, since every possible complex $\varepsilon$ of the passive medium (with $\operatorname{Im}[\varepsilon]<0$ ) has been checked, the upper limit for absorption by homogeneous structures concerns also all layers thinner than our metasurface uniform layers (thicknesses between 0 and $2 a$ ).

\section{CONCLUDING REMARKS}

A family of metasurfaces comprising periodically corrugated parallel rods consisting of two media was considered. Such a class of particles offers the possibility of controlling the electromagnetic characteristics of their external surface while keeping a smooth textural transition. We converted the configuration to an equivalent multilayered one (with the use of an effective-medium approximation), which is analytically solvable. Sharp changes in the transmissivity and the absorbance of the device were reported when it was operated in the regime of triggering a new refraction order. Such intriguing effects can be directly attributed to the lattice resonances, namely, the constructive or destructive interference of scattering from consecutive particles.

It should be stressed that the proposed designs are not fully optimized; several conventional parametric choices have been made. Therefore, there is a significant margin for further calibration by greatly varying the rest of the size and texture features or by demanding different constituent properties for the external surface of the particles. Given the fact that our design supports analytical solutions and semianalytical approximations, there is no need for extensive cumbersome fullwave simulations via commercial software for such a global optimization. Therefore, the technique and the assumptions of the present work can be easily expanded to give more general and improved metasurfaces, revealing even more fascinating modes of operation at low computational cost.

\section{ACKNOWLEDGMENTS}

The authors would like to thank Prof. S. Tretyakov (Aalto University, Finland) for useful discussions on bipolar approximation in metasurfaces. This work was partially supported by Nazarbayev University Small Grants with the project entitled "Super transmitters, radiators and lenses via photonic synthetic matter" (Grant No. 090118FD5349). Funding from MES RK state-targeted program BR05236454 is also acknowledged. 


\section{APPENDIX: FORMULAS AND \\ COMMENTS ON DERIVATIONS}

\section{Derivation of formula (4) from formula (3)}

The addition theorem for cylindrical coordinates of a parallel (common) $z$ axis [30] connects the eigenfunctions of the cylindrical Helmholtz equation in one coordinate system $\left(r_{n}, \varphi_{n}\right)$ to the other $(r, \varphi)$. In particular, if the angle of the origin $O_{n}$ of the local system in terms of the global one equals $\pm \pi / 2$ (in proportion to the sign of $n$ ), the addition theorem gives (apparently for $n \neq 0$ because the $n=0$ local coordinate system of the central cylinder coincides with the global one)

$$
\begin{aligned}
H_{0}^{(2)}\left(k_{0} r_{n}\right) & =\sum_{p=-\infty}^{+\infty} H_{p}^{(2)}\left(2 k_{0} b|n|\right) J_{p}\left(k_{0} r\right) j^{p}\left[L_{p}^{+} \cos (p \varphi)-j \operatorname{sgn}(n) L_{p}^{-} \sin (p \varphi)\right], \\
H_{1}^{(2)}\left(k_{0} r_{n}\right) \cos \varphi_{n} & =\sum_{p=-\infty}^{+\infty} H_{p-1}^{(2)}\left(2 k_{0} b|n|\right) J_{p}\left(k_{0} r\right) j^{p}\left[-j L_{p-1}^{+} \cos (p \varphi)-\operatorname{sgn}(n) L_{p-1}^{-} \sin (p \varphi)\right], \\
H_{1}^{(2)}\left(k_{0} r_{n}\right) \sin \varphi_{n} & =\sum_{p=-\infty}^{+\infty} H_{p-1}^{(2)}\left(2 k_{0} b|n|\right) J_{p}\left(k_{0} r\right) j^{p}\left[\operatorname{sgn}(n) L_{p-1}^{-} \cos (p \varphi)-j L_{p-1}^{+} \sin (p \varphi)\right],
\end{aligned}
$$

where $L_{p}^{ \pm}=\frac{1 \pm(-1)^{p}}{2}$.

By keeping from the sums (A1), (A2), and (A3) only the first two orders $p=0, \pm 1$ and by acting with the operator $\mathcal{J}\{\star\}=\sum_{n=-\infty}^{+\infty}\{\star\}$ of (3) only to $n$-dependent quantities (not those solely dependent on global $r$ and $\varphi$ ), we obtain (4). The sums $\Sigma_{0}, \Sigma_{1}, \Sigma_{2}$ are defined as

$$
\begin{gathered}
\Sigma_{0}=\sum_{n=-\infty}^{+\infty} e^{-2 j k_{0} b n \sin \theta} H_{0}^{(2)}\left(2 k_{0} b|n|\right), \\
\Sigma_{1}=\sum_{n=-\infty}^{+\infty} e^{-2 j k_{0} b n \sin \theta} H_{1}^{(2)}\left(2 k_{0} b|n|\right) \operatorname{sgn}(n), \\
\Sigma_{2}=\sum_{n=-\infty}^{+\infty} e^{-2 j k_{0} b n \sin \theta} H_{2}^{(2)}\left(2 k_{0} b|n|\right),
\end{gathered}
$$

where the primed sums exclude the term $n=0$.

\section{Numerical evaluation of series (A4), (A5), and (A6)}

The series $\Sigma_{0}, \Sigma_{1}, \Sigma_{2}$ of (A4), (A5), and (A6) respectively are very slowly convergent with a general term behaving like $\mathrm{O}\left(e^{-2 j k_{0} b(|n|+n \sin \theta)} / \sqrt{|n|}\right)$ for $|n| \rightarrow+\infty$. Therefore, directly evaluating them is not efficient. To overcome such a computational snag, we exploit the analytical formula [42]

$$
\sum_{n=1}^{+\infty} \frac{e^{-2 j k_{0} b(|n|+n \sin \theta)}}{\sqrt{|n|}}=\operatorname{Li}_{\frac{1}{2}}\left(e^{-2 j k_{0} b(1+\sin \theta)}\right),
$$

where $\mathrm{Li}_{v}$ is the polylogarithm function of order $v$. In particular, we subtract the asymptotic general terms from the corresponding series (A4), (A5), and (A6), and we simultaneously add the aforementioned equivalent analytical result. The remaining sum converges much faster since the higher-order terms of the difference vanish rapidly.

\section{Derivation of formula (5) from formula (3)}

By applying laws of cosines and sines in the triangle with vertices: the global origin $O$, the local origin $O_{n}$ (centralized at the $n$th cylinder), and the observation point $(x, y)$, we readily find

$$
\begin{aligned}
\cos \varphi_{n} & =\frac{x}{\sqrt{x^{2}+(y-2 b n)^{2}}}, \\
\sin \varphi_{n} & =\frac{y-2 b n}{\sqrt{x^{2}+(y-2 b n)^{2}}} .
\end{aligned}
$$

Given the fact that $r_{n}=r_{n}(x, y)=\sqrt{x^{2}+(y-2 b n)^{2}}$, we can write the scattering field of (3) as follows:

$$
G_{\text {scat }}=S_{0} Q-\frac{S_{1 c} Q_{x}+S_{1 s} Q_{y}}{k_{0}},
$$

where the subscripts $x, y$ correspond to partial derivatives of the function $Q=Q(x, y)$, which is defined as a series,

$$
Q=\sum_{n=-\infty}^{+\infty} e^{-2 j k_{0} b n \sin \theta} H_{0}^{(2)}\left(k_{0} r_{n}(x, y)\right) .
$$

The function $Q$ can be transformed with using the Poisson summation formula [34] and the following integral representation of the Hankel function [34]:

$$
\begin{aligned}
& H_{0}^{(2)}\left(k_{0} \sqrt{(x-X)^{2}+(y-Y)^{2}}\right) \\
& =\frac{j}{\pi} \int_{-\infty}^{+\infty} \frac{e^{-j \beta(y-Y)-|x-X| \sqrt{\beta^{2}-k_{0}^{2}}}}{\sqrt{\beta^{2}-k_{0}^{2}}} d \beta .
\end{aligned}
$$

More specifically,

$$
Q=j e^{-j k_{0} y \sin \theta} \sum_{m=-\infty}^{+\infty} \frac{e^{-\kappa_{m}|x| / b-j m \pi y / b}}{\kappa_{m}},
$$

where $\kappa_{m}=\sqrt{\left(m \pi+k_{0} b \sin \theta\right)^{2}-\left(k_{0} b\right)^{2}}$. If one substitutes (A13) in (A10), one obtains (5). 
[1] C. L. Holloway, E. F. Kuester, J. A. Gordon, J. O'Hara, J. Booth, and D. R. Smith, An overview of the theory and applications of metasurfaces: The two-dimensional equivalents of metamaterials, IEEE Antennas Propag. Mag. 54, 10 (2012).

[2] N. Yu and F. Capasso, Flat optics with designer metasurfaces, Nat. Mater. 13, 139 (2014).

[3] S. B. Glybovski, S. A. Tretyakov, P. A. Belov, Y. S. Kivshar, and C. R. Simovski, Metasurfaces: From microwaves to visible, Phys. Rep. 634, 1 (2016).

[4] J. Li, C. Shen, A. Diaz-Rubio, S. A. Tretyakov, and S. A. Cummer, Systematic design and experimental demonstration of bianisotropic metasurfaces for scattering-free manipulation of acoustic wavefronts, Nat. Commun. 9, 1342 (2018).

[5] N. Yu, P. Genevet, M. A. Kats, F. Aieta, J.-P. Tetienne, F. Capasso, and Z. Gaburro, Light propagation with phase discontinuities: Generalized laws of reflection and refraction, Science 334, 333 (2011).

[6] A. V. Kildishev, A. Boltasseva, and V. M. Shalaev, Planar photonics with metasurfaces, Science 339, 1232009 (2013).

[7] Y. Zhao and A. Alu, Manipulating light polarization with ultrathin plasmonic metasurfaces, Phys. Rev. B 84, 205428 (2011).

[8] A. Arbabi, Y. Horie, M. Bagheri, and A. Faraon, Dielectric metasurfaces for complete control of phase and polarization with subwavelength spatial resolution and high transmission, Nat. Nanotechnol. 10, 937 (2015).

[9] D. Lin, P. Fan, E. Hasman, and M. L. Brongersma, Dielectric gradient metasurface optical elements, Science 345, 298 (2014).

[10] X. Ni, Z. J. Wong, M. Mrejen, Y. Wang, and X. Zhang, An ultrathin invisibility skin cloak for visible light, Science 349, 1310 (2015).

[11] V. S. Asadchy, A. Diaz-Rubio, S. N. Tcvetkova, D. H. Kwon, A. Elsakka, M. Albooyeh, and S. A. Tretyakov, Flat Engineered Multichannel Reflectors, Phys. Rev. X 7, 031046 (2017).

[12] J. D. Joannopoulos, S. G. Johnson, J. N. Winn, and R. D. Meade, Photonic Crystals: Molding the Flow of Light (Princeton University Press, Princeton, NJ, 2011).

[13] H. J. Lezec, A. Degiron, E. Devaux, R. A. Linke, L. MartinMoreno, F. J. Garcia-Vidal, and T. W. Ebbesen, Beaming light from a subwavelength aperture, Science 297, 820 (2002).

[14] N. L. Tsitsas and C. A. Valagiannopoulos, Anomalous reflection of visible light by all-dielectric gradient metasurfaces, J. Opt. Soc. Am. B 34, D1 (2017).

[15] C. A. Valagiannopoulos and S. A. Tretyakov, Symmetric absorbers realized as gratings of PEC cylinders covered by ordinary dielectrics, IEEE Trans. Antennas Propag. 62, 5089 (2014).

[16] K. T. Fountaine, W. S. Whitney, and H. A. Atwater, Resonant absorption in semiconductor nanowires and nanowire arrays: Relating leaky waveguide modes to Bloch photonic crystal modes, J. Appl. Phys. 116, 153106 (2014).

[17] A. P. Slobozhanyuk, A. N. Poddubny, A. J. Raaijmakers, C. A. van den Berg, A. V. Kozachenko, I. A. Dubrovina, I. V. Melchakova, Y. S. Kivshar, and P. A. Belov, Enhancement of magnetic resonance imaging with metasurfaces, Adv. Mater. 28, 1832 (2016).
[18] D. Ye, L. Lu, J. D. Joannopoulos, M. Soljacic, and L. Ran, Invisible metallic mesh, Proc. Natl. Acad. Sci. USA 113, 2568 (2016).

[19] V. O. Byelobrov, T. L. Zinenko, K. Kobayashi, and A. I. Nosich, Periodicity matters: Grating or lattice resonances in the scattering by sparse arrays of subwavelength strips and wires, IEEE Antennas Propag. Mag. 57, 34 (2015).

[20] V. Twersky, On scattering of waves by the infinite grating of circular cylinders, IRE Trans. Antennas Propag. 10, 737 (1962).

[21] K. Yasumoto, H. Toyama, and T. Kushta, Accurate analysis of two-dimensional electromagnetic scattering from multilayered periodic arrays of circular cylinders using lattice sums technique, IEEE Trans. Antennas Propag. 52, 2603 (2004).

[22] C. Li, D. Lesselier, and Y. Zhong, Scattering of obliquely incident electromagnetic plane waves by composite panel involving periodic arrays of circular fibers, IEEE Trans. Antennas Propag. 63, 3168 (2015).

[23] Ö. Kavaklioğlua and B. Schneider, On multiple scattering of radiation by an infinite grating of dielectric circular cylinders at oblique incidence, Int. J. Infrared Millimeter Waves 29, 329 (2008).

[24] J. P. Kottmann and O. J. F. Martin, Accurate solution of the volume integral equation for high-permittivity scatterers, IEEE Trans. Antennas Propag. 48, 1719 (2000).

[25] C. Della Giovampaola and N. Engheta, Digital metamaterials, Nat. Mater. 13, 1115 (2014).

[26] A. H. Sihvola, Electromagnetic Mixing Formulas and Applications, IEE Electromagnetic Waves Series Vol. 47 (IEEE Press, London, 1999).

[27] C. A. Valagiannopoulos and P. Alitalo, Electromagnetic cloaking of cylindrical objects by multilayer or uniform dielectric claddings, Phys. Rev. B 85, 115402 (2012).

[28] W. Lu, D. Wang, and L. Chen, Near-static dielectric polarization of individual carbon nanotubes, Nano Lett. 7, 2729 (2007).

[29] J. Venermo and A. Sihvola, Dielectric polarizability of circular cylinder, J. Electrost. 63, 101 (2005).

[30] J. A. Stratton, Electromagnetic Theory (McGraw-Hill, New York, 1941), Chap. 6, pp. 372-374.

[31] R. Petit, Electromagnetic Theory of Gratings, Topics in Current Physics Vol. 22 (Springer, Berlin, 1980), p. 38.

[32] C. A. Valagiannopoulos and N. L. Tsitsas, Linearization of the T-matrix solution for quasi-homogeneous scatterers, J. Opt. Soc. Am. A 26, 870 (2009).

[33] C. A. Balanis, Advanced Engineering Electromagnetics (Wiley, New York, 1989), p. 596.

[34] P. M. Morse and H. Feshbach, Methods of Theoretical Physics, Part I, International Series in Pure and Applied Physics (McGraw-Hill Book Company, New York, 1953), pp. 467, 823.

[35] C. F. Bohren and D. R. Huffman, Absorption and Scattering of Light by Small Particles (Wiley-VCH, New York, 2004), p. 327.

[36] W. L. Barnes, Particle Plasmons: Why Shape Matters, Am. J. Phys. 84, 593 (2016).

[37] X. Xia, J. Tu, Y. Zhang, X. Wang, C. Gu, X. Zhao, and H. J. Fan, High-quality metal oxide core/shell nanowire arrays on conductive substrates for electrochemical energy storage, ACS Nano 6, 5531 (2012). 
[38] X. Shena, T. J. Cuia, D. Martin-Canob, and F. J. GarciaVidal, Conformal surface plasmons propagating on ultrathin and flexible films, Proc. Natl. Acad. Sci. USA 110, 40 (2013).

[39] Y. Radi, C. R. Simovski, and S. A. Tretyakov, Thin Perfect Absorbers for Electromagnetic Waves: Theory, Design, and Realizations, Phys. Rev. Appl. 3, 037001 (2015).
[40] A. N. Papadimopoulos, N. V. Kantartzis, N. L. Tsitsas, and C. A. Valagiannopoulos, Wide-angle absorption of visible light from simple bilayers, Appl. Opt. 56, 9779 (2017).

[41] Refractive index database, http://refractiveindex.info/.

[42] L. Lewin, Structural Properties of Polylogarithms, Mathematical Surveys and Monographs (American Mathematical Society, New York, 1991), p. 6. 